\title{
Study on One-cycle Control Three-phase Four-wire APF with Four-leg Topology
}

\author{
Hanying Gao, Hairui Wang, Duanzeng Liu and Weili Li \\ School of Electrical and Electronic Engineering, \\ Harbin University of Science and Technology, Harbin, China \\ E-mail: ghyljt@sina.com
}

\begin{abstract}
Proposes a three-phase four-wire APF with four-leg topology based on one-cycle control. Main circuit adopts three-phase four-leg power topology. The fourth bridge arm connects to the zero line, which can inhibit the system zero sequence current. This topology is suitable for high power balanced and unbalanced load. Applying one-cycle control strategy, compares with the traditional control method of APF, it has advantages, such as fast dynamic response, strong anti-interference ability and so on. Meanwhile, the circuit structure is simple, low cost and easy to implement. Simulation and experimental results show one-cycle control with three-phase four-leg APF can effectively compensate harmonic, zero sequence and reactive current.
\end{abstract}

Keywords: three-phase four-wire, four-leg, APF, one-cycle control

\section{Introduction}

With the more and more widespread use of power electronics technology, it has benefited a lot to the grid, meanwhile has brought a lot of disadvantages. Due to nonlinearity of the power electronics equipment, the majority of them share relatively low power factor, and easily generate harmonics at work, what' s more, there are a large number of impact and low power factor loads in grid, which seriously affect the power quality $[1,2]$. To restrain the system harmonic and compensate system reactive power effectively, APF is one of the key technologies.

In recent years, research on APF is becoming more and more perfect, but usually utilizes traditional control methods, such as theory of the instantaneous reactive power and that of FFT distortion current detection, etc [3]. In order to eliminate deviation, the linear feedback is adopted through controlling duty cycle of the power switch. Although this method is effective, it has disadvantages, such as complex structure, high cost and poor anti-interference ability, etc [4].

One cycle control is a nonlinear control technology, which was first proposed by American scholars [5]. This method has constant switch frequency, and can make average value of the switching time be proportional to the given value in a switching period, thus, eliminates the steady-state and transient error [6-8].

With no multiplier circuit and fewer sampling variables, the control system has the advantages of simple structure, low cost, fast response and strong anti-interference ability [9].

In this paper one-cycle control technique is applied in the APF, and one-cycle control with a three-phase four-leg topology is proposed, the system is suitable for high power balanced and unbalanced load, which realize an effective compensation for system harmonic, zero-sequence and reactive current. 


\section{Theoretical Analysis of One-cycle Control Three-phase APF with Four Leg}

Figure 1 shows one-cycle control system with grid side current detection circuit, DC side capacitor voltage detection circuit, one cycle control unit, IGBT drive unit and a three-phase four-leg inverter unit. Through taking the circuit outside the grid as pure resistance circuit, modeling based on circuit structure, deducing the control equation and selecting reasonable parameters, we can realize APF with one-cycle control strategy.

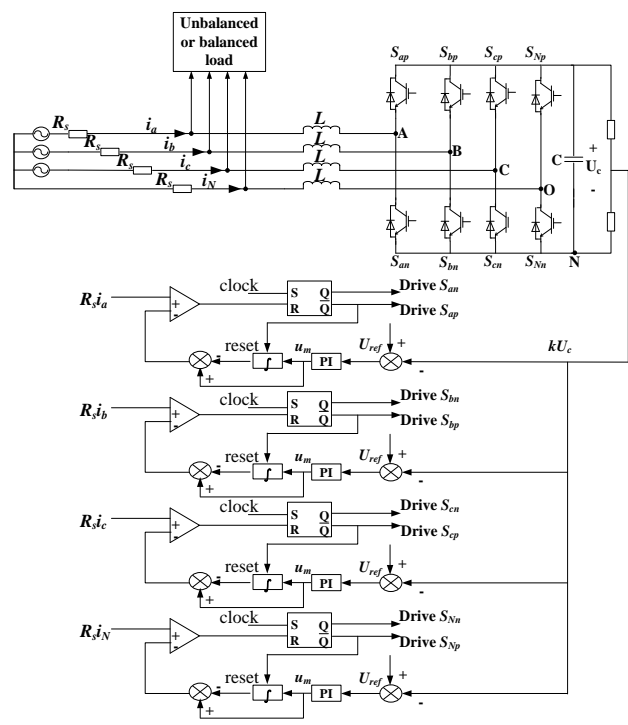

Figure 1. Schematic Diagram of One-cycle Control with Three-phase Four-leg APF

Assuming inverter unit is working under the continuous current mode, three-phase system is symmetric and the switching frequency is much higher than the grid frequency, the average voltage of nodes $\mathrm{A}, \mathrm{B}, \mathrm{C}$ and $\mathrm{O}$ to negative terminal node $\mathrm{N}$ of $\mathrm{DC}$ side within each switching period can be written as

$$
\left\{\begin{array}{l}
u_{A N}=\left(1-D_{a n}\right) U_{c} \\
u_{B N}=\left(1-D_{\mathrm{b} n}\right) U_{c} \\
u_{C N}=\left(1-D_{c n}\right) U_{c} \\
u_{O N}=\left(1-D_{o n}\right) U_{c}
\end{array}\right.
$$

where $D_{a n}, D_{b n}, D_{c n}$, and $D_{o n}$ are duty cycle of lower-leg switches $S_{a n}, S_{b n}, S_{c n}$ and $S_{o n}$ respectively. Figure 2 shows the average model of one-cycle control with three-phase four-leg APF [10].

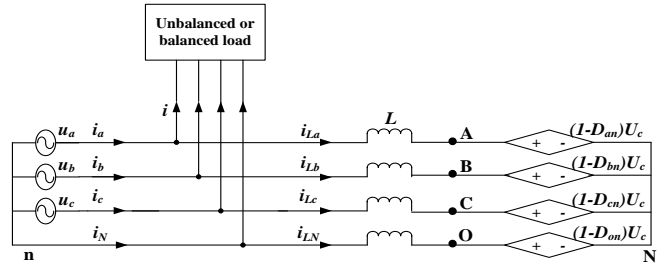

Figure 2. Average Model of One-cycle Control with Three-phase Four-leg APF 
From formula (1), the average voltage of nodes A, B and C to $\mathrm{O}$ can be derived as

$$
\left\{\begin{array}{l}
u_{A O}=\left(D_{o n}-D_{a n}\right) U_{c} \\
u_{B O}=\left(D_{o n}-D_{b n}\right) U_{c} \\
u_{C O}=\left(D_{o n}-D_{c n}\right) U_{c}
\end{array}\right.
$$

Average voltage vectors of nodes $\mathrm{A}, \mathrm{B}$ and $\mathrm{C}$ to $\mathrm{O}$ equal to the three-phase voltage vectors minus the voltage drop across inverter inductors. Here

$$
\left\{\begin{array}{l}
\mathbf{u}_{\mathbf{A O}}=\mathbf{u}_{\mathbf{a}}-j \omega L \mathbf{i}_{\mathbf{L a}}+j \omega L \mathbf{i}_{\mathbf{L N}} \\
\mathbf{u}_{\mathbf{B O}}=\mathbf{u}_{\mathbf{b}}-j \omega L \mathbf{i}_{\mathbf{L b}}+j \omega L \mathbf{i}_{\mathbf{L N}} \\
\mathbf{u}_{\mathbf{C O}}=\mathbf{u}_{\mathbf{c}}-j \omega L \mathbf{i}_{\mathbf{L c}}+j \omega L \mathbf{i}_{\mathbf{L N}}
\end{array}\right.
$$

where $\mathrm{L}$ represents the inductance connecting to the grid (assuming four connecting inductance values are equal), $\omega$ is angular frequency of the grid.

Because the inductor is in the high-frequency circuit, the inductance value of grid side is very small. Since the voltage drop across grid connecting inductance is much smaller than the phase voltage value, it can be ignored, formula (3) can be simplified as

$$
\left\{\begin{array}{l}
u_{A O} \approx u_{a} \\
u_{B O} \approx u_{b} \\
u_{C O} \approx u_{c}
\end{array}\right.
$$

From formulae (2) and (4), we get

$$
\left\{\begin{array}{l}
u_{a}=\left(D_{o n}-D_{a n}\right) U_{c} \\
u_{b}=\left(D_{o n}-D_{b n}\right) U_{c} \\
u_{c}=\left(D_{o n}-D_{c n}\right) U_{c}
\end{array}\right.
$$

For three-phase symmetric systems, there is

$$
u_{a}+u_{b}+u_{c}=0
$$

From formulae (5) and (6), we get

$$
D_{o n}=\frac{1}{3}\left(D_{a n}+D_{b n}+D_{c n}\right)
$$

Substituting formula (7) into formula (5), we can get

$$
\left[\begin{array}{ccc}
-\frac{2}{3} & \frac{1}{3} & \frac{1}{3} \\
\frac{1}{3} & -\frac{2}{3} & \frac{1}{3} \\
\frac{1}{3} & \frac{1}{3} & -\frac{2}{3}
\end{array}\right] \cdot\left[\begin{array}{c}
D_{a n} \\
D_{b n} \\
D_{c n}
\end{array}\right]=\frac{1}{U_{c}}\left[\begin{array}{l}
u_{a} \\
u_{b} \\
u_{c}
\end{array}\right]
$$

Those formulae provide relationships between duty cycle of three-phase power switches and three-phase voltage. Because the formula coefficient matrix is a singular matrix with infinitely many solutions, those formulae can be assumed as 


$$
\left\{\begin{array}{l}
D_{a n}=K_{1}+K_{2} \frac{u_{a}}{U_{c}} \\
D_{b n}=K_{1}+K_{2} \frac{u_{b}}{U_{c}} \\
D_{c n}=K_{1}+K_{2} \frac{u_{c}}{U_{c}}
\end{array}\right.
$$

Substitute formula (9) into formula (8), here $K_{2}=-1$ and $K_{1}$ can take any value,thus

$$
\left\{\begin{array}{l}
D_{a n}=K_{1}-\frac{u_{a}}{U_{c}} \\
D_{b n}=K_{1}-\frac{u_{b}}{U_{c}} \\
D_{c n}=K_{1}-\frac{u_{c}}{U_{c}}
\end{array}\right.
$$

To achieve unit power factor, regard circuits outside the grid as a pure resistance load [11], which satisfies

$$
\left\{\begin{array}{l}
u_{a}=R_{e} i_{a} \\
u_{b}=R_{e} i_{b} \\
u_{c}=R_{e} i_{c}
\end{array}\right.
$$

where $R_{e}$ is the equivalent resistance of each phase.

Substituting formula (11) into the formula (10), multiplying by current sampling resistor $R_{s}$ on both sides of formulae, and taking $R_{s} K_{l} U_{c} / R_{e}$ as $u_{m}$, combining with formula (7), we can obtain

$$
\left\{\begin{array}{c}
R_{s} i_{a}=u_{m}-D_{a n} u_{m} / K_{1} \\
R_{s} i_{\mathrm{b}}=u_{m}-D_{b n} u_{m} / K_{1} \\
R_{s} i_{c}=u_{m}-D_{c n} u_{m} / K_{1} \\
R_{s} i_{N}=3\left(u_{m}-D_{o n} u_{m} / K_{1}\right)
\end{array}\right.
$$

Within each switching period, current and voltage um can be approximately regarded as constant values. After equation (12) are integrated in one switching period, the following equations can be obtained

$$
\left\{\begin{array}{c}
R_{s} i_{a}=u_{m}-1 / T_{i} \int_{0}^{D_{a n} T_{s}} u_{m} d t \\
R_{s} i_{\mathrm{b}}=u_{m}-1 / T_{i} \int_{0}^{D_{b n} T_{s}} u_{m} d t \\
R_{s} i_{c}=u_{m}-1 / T_{i} \int_{0}^{D_{c n} T_{s}} u_{m} d t \\
R_{s} i_{N}=3\left(u_{m}-1 / T_{i} \int_{0}^{D_{o n} T_{s}} u_{m} d t\right)
\end{array}\right.
$$

where $T_{s}$ is the switching period, $T_{i}$ is the constant of integration and $T_{i}=K_{l} T_{s}$.

Formula (13) is the key formula to realize one-cycle control strategy [12]. Since the duty cycle is from 0 to 1 , substitute $K_{l}=T_{i} / T_{s}$ into formula (10), we get 


$$
D_{a n}=K_{1}-\frac{u_{a}}{U_{c}}=\frac{T_{i}}{T_{s}}-\frac{u_{a}}{U_{c}}
$$

Due to $0<D_{a n}<1$, the following formula can be obtained

$$
\frac{u_{a}}{U_{c}}<\frac{T_{i}}{T_{s}}<1+\frac{u_{a}}{U_{c}}
$$

Because $u_{a}$ is a sine wave, limit values of the above formula should be taken as the boundary conditions [13], namely

$$
\frac{u_{a \max }}{U_{c}}<\frac{T_{i}}{T_{s}}<1+\frac{u_{a \min }}{U_{c}}
$$

where $u_{\text {amax }}$ is the peak value of sine wave, which is approximately $311 \mathrm{~V}, \quad u_{\text {amin }}$ is the valley value of sine wave, which is approximately $-311 \mathrm{~V}$, pre-charge voltage $U_{c}$ of the capacitor is $800 \mathrm{~V}$, thus $0.4 T_{s}<T_{i}<0.6 T_{s}$

Simulation results show that the compensation effect is the best with $T_{i}$ equals $0.5 T_{s}$, so the ultimate control formula can be derived as

$$
\left\{\begin{array}{c}
R_{s} i_{a}=u_{m}\left(1-2 D_{a n}\right) \\
R_{s} i_{\mathrm{b}}=u_{m}\left(1-2 D_{b n}\right) \\
R_{s} i_{c}=u_{m}\left(1-2 D_{c n}\right) \\
R_{s} i_{N}=3 u_{m}\left(1-2 D_{o n}\right)
\end{array}\right.
$$

Formula (17) describes numerical relations among the grid current, the duty cycle and output of the voltage regulator, which is the target formula of one-cycle control. Therefore, according to formula (13), it can be seen, when the formula (17) is satisfied by switch duty cycle, output of the voltage regulator, three-phase and zero line current, APF can be realized effectively.

According to the formula (17) and (13), one cycle control unit consists of the integrator with reset function, PI regulator, RS trigger and a voltage comparator, as shown in Figure 3. From Figure 3, the clock circuit generates a constant frequency clock signal to $S$ terminal of the RS trigger, period is $T_{s}$. Within a switching period $T_{s}$, each pair of power switches within four legs are complementary, duty cycle of lower and upper leg $S_{n}, S_{p}$ are $D_{n}, 1-D_{n}$ respectively. Through sampling circuit, voltage $R_{s} i_{s}$ and $k U_{c}$ are input to the onecycle controller, which are positive input terminal of the voltage comparator and reverse input of the PI regulator respectively. With deviation between the DC sampling voltage $k U_{c}$ and the reference voltage value $U_{\text {ref }}$ being input to the PI regulator, and then PI adjustment being conducted, integral output $u_{m}$ can be obtained. From the $t_{0}$ moment, the $\mathrm{S}$ terminal of RS trigger is at the high level, the lower leg $S_{n}$ is on, the upper leg $S_{p}$ is off, $i_{s}$ raises. Meanwhile, resettable integrator starts, conduct integral to the PI regulator output value $u_{m}$, the integral quantity $1 / T_{i} \int_{0}^{t} u_{m} d t$ is obtained, where $T_{i}=0.5 T_{s}$. The deviation between output value of PI regulator and integral value, namely $u_{m}-1 / T_{i} \int_{0}^{t} u_{m} d t$ is input to the reverse terminal of voltage comparator, then compared with the product of $R_{s}$ and $i_{s}$. At the $t_{l}$ moment $i_{s}$ increased to make the product of $R_{s}$ and $i_{s}$ more than $u_{m}-1 / T_{i} \int_{0}^{t} u_{m} d t$, the integral time is $D_{n} T_{s}$, which satisfies the formula $R_{s} i_{s}=U_{m}\left(1-2 D_{n}\right)$. A high level from the comparator will be input to the R terminal of RS trigger, Q terminal outputs a low level, then the lower leg $S_{n}$ is off, the upper leg $S_{p}$ is on, $i_{s}$ decreased, meanwhile, the $\overline{\mathrm{Q}}$ terminal is at the high level, enables reset of the integrator, till the $t_{2}$ moment on the rising edge of $\mathrm{S}$ clock arriving, this cycle terminates and starts repetitive action of the last cycle. Each phase of the three-phase and neutral line should utilize an independent one-cycle control, the only difference lies in that integration value of the one-cycle controller for every 
single phase within three phases is $u_{m}$ while integration value of the one-cycle controller for neutral line is $3 u_{m}$. Four one-cycle controllers repeat the above actions simultaneously, which enables grid current to track the grid voltage rapidly and accurately, which will compensate grid harmonic, zero-sequence current and reactive current effectively. To prevent two power switches of the same leg turn on simultaneously, we should add deadzone circuit, which brings dead time into the complementary drive waveform of upper and lower legs.

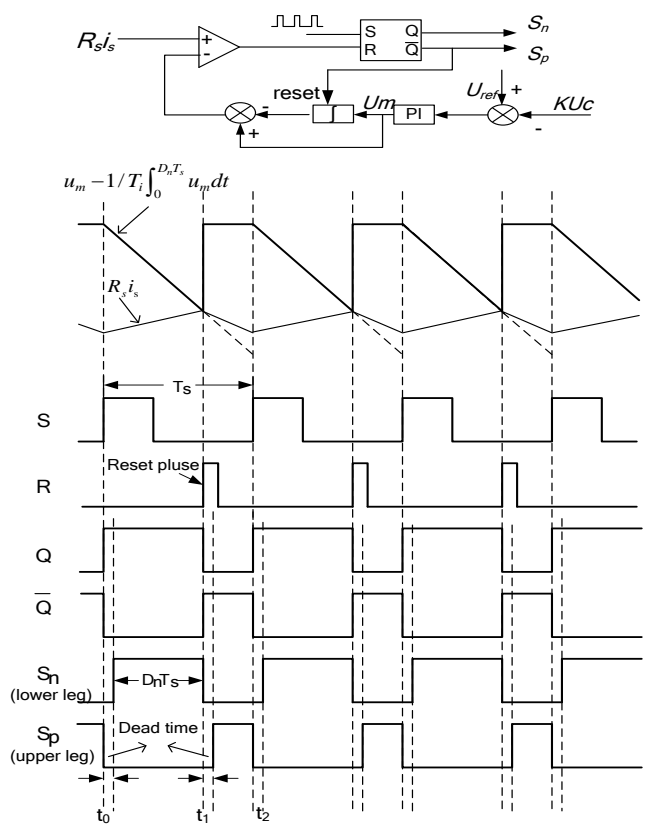

Figure 3. Single-phase Equivalent Model of One-cycle Controller

\section{The Design of Hardware Circuit}

\subsection{Detection Circuit and Signal Processing Circuit}

Grid current and DC side voltage should be sampled during the one-cycle control process. From Figure 4, the grid current sampling current adopts the linear optocoupler HCPL7840, the sampling resistors connects three-phase and neutral line, voltage across the sampling resistor inputs to 2,3 pin of the HCPL7840, we can obtain a $-1.6 \mathrm{~V} \sim+1.6 \mathrm{~V}$ differential voltage signal in the secondary side. When the output differential signal of HCPL7840 is amplified, properly processed from input differential signal to the unipolar signal and input to reverse input terminal of the voltage comparator, the current sampling signal can be obtained in Figure 5 [14].

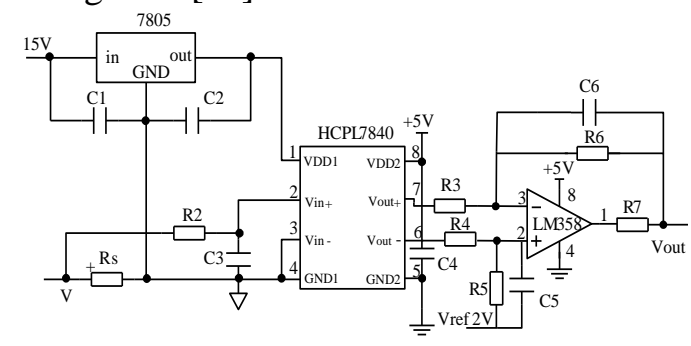

Figure 4. Grid Current Sampling Circuit with Linear Optocoupler

In order to realize the bidirectional energy flow between DC side of converter and the grid, the DC side voltage must be higher than the peak value of line voltage, therefore, the 
reference voltage of DC side is selected as $800 \mathrm{~V}$. Figure 6 shows voltage sampling circuit of the DC side, here voltage hall sensor is adopted and type of voltage hall sensor is CHV-25P. According to the reference control voltage $U_{\text {ref }}$, resistance values on both sides of the sensor can be determined and the sample value $k U_{c}$ can be obtained.

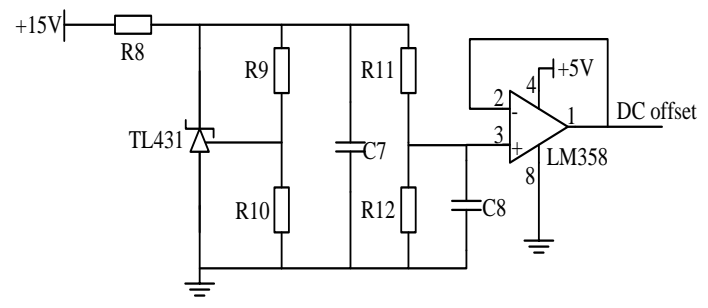

Figure 5. Offset Voltage Circuit

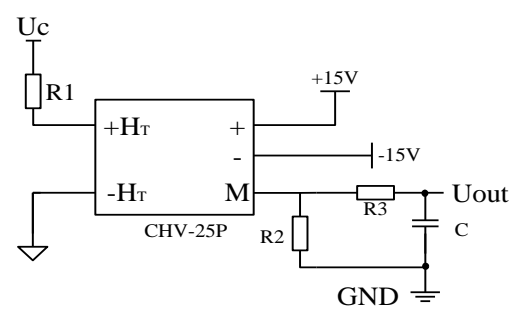

Figure 6. Voltage Sampling Circuit of DC Side

\subsection{One-cycle Control Circuit}

One-cycle control circuit consists of the integrator with reset function, PI regulator, RS trigger and a voltage comparator. Figure 7 shows integrator consists of a integral circuit with LM358 and CD4066. When Q terminal of the RS trigger outputs low level, the analog switch is off, the integrator conduct integral to the input signal, however when the $\overline{\mathrm{Q}}$ terminal outputs high level, the analog switch is on, the capacitor $\mathrm{C}$ is grounded and integrator is reset. Since the analog switch is not an ideal component and with certain resistance, which will limit the current on the switch being closed [15].

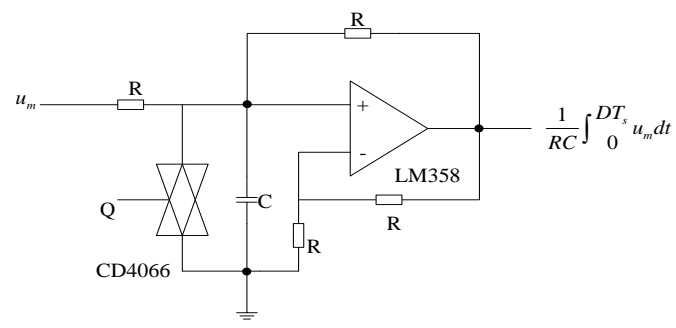

Figure 7. Integrator with Reset Function

To ordinary RS trigger, the output varies if $\mathrm{RS}=1$. which should be avoided, especially in the one-cycle control, $\mathrm{Q}$ and $\overline{\mathrm{Q}}$ of the RS trigger are adopted to generate complementary driving signals to power switches of the same leg, if uncertain situation of output appears, it may possibly occur shoot-through fault. As shown in Figure 8, in order to avoid this situation occurring, the circuit consists of 74LS74 and gate circuits, which avoid occurrence of the uncertain output. 


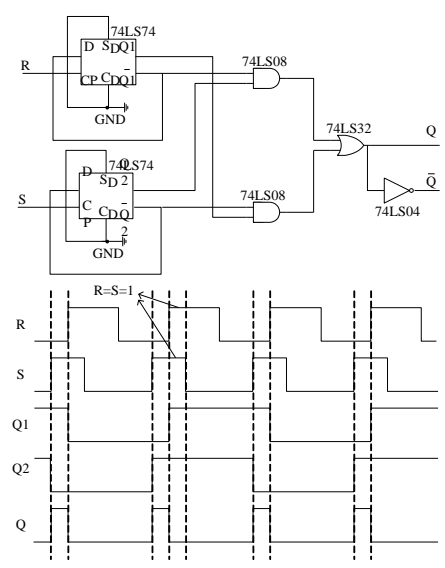

\section{Figure 8. RS Trigger and Sequential Chart}

\subsection{Driving Circuit}

To prevent occurrence of the shoot-through fault, add dead zone circuit in Figure 9, here, $\mathrm{RC}$ forms a delay circuit. When rising edge of $\mathrm{Q}$ or $\overline{\mathrm{Q}}$ arrives, voltage on the capacitor $\mathrm{C}$ rises continuously, when rising to a threshold voltage, A or B outputs high level, so it generates a certain delay time in their rising edge respectively. By shaping and amplifying circuit, we can obtain driving signals of upper and lower legs.

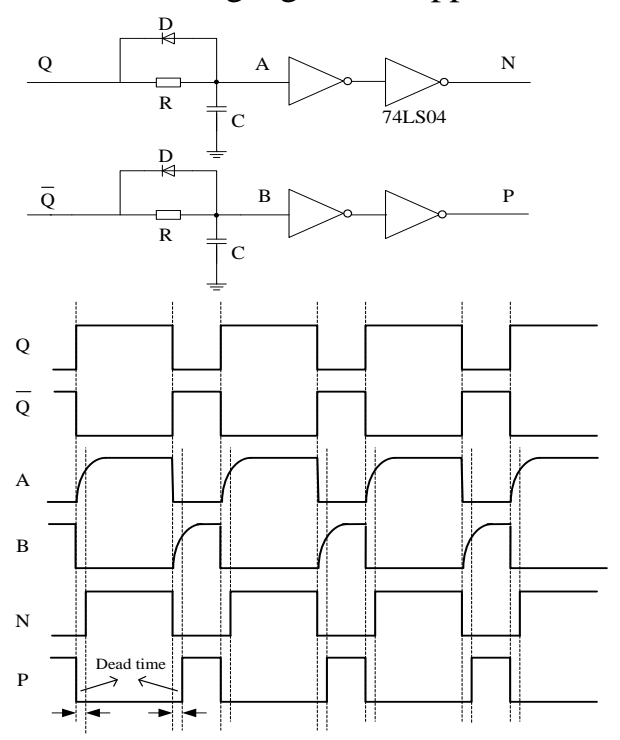

Figure 9. Dead-zone Circuit and Waveform Analysis

Because IGBT has many advantages of fast switching speed, low conductive resistance and high withstand voltage and so on, it is adopted as the power switch. Because TLP250 has many advantages, such as isolation between input and output, wide voltage range, fast switching speed, driving capability with $0.5 \mathrm{~A}$, withstand voltage with $1200 \mathrm{~V}$ and so on, it is chosen to drive power switch in Figure 10. 


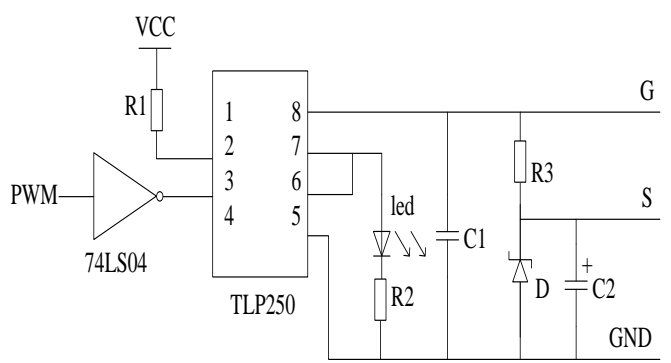

Figure 10. Driving Circuit

\section{Simulation Research}

In this paper, Matlablsimulink simulation is adopted to verify feasibility of the onecycle control strategy under two kinds of load. The main parameters are as follows: the switching frequency is $20 \mathrm{kHz}$, the DC side voltage is $800 \mathrm{~V}$ and inductance $\mathrm{L}$ with the three-phase four-leg topology is $1 \mathrm{mH}$.

(1) Three-phase unbalanced nonlinear loads: phase A is connected with single phase rectifier with a resistance-inductance load $(\mathrm{R}=30 \Omega, \mathrm{L}=50 \mathrm{mH})$, phase $\mathrm{B}$ is connected with a resistance load $(\mathrm{R}=5 \Omega)$, phase $\mathrm{C}$ is connected with a resistance-inductance load $(\mathrm{R}=10 \Omega, \mathrm{L}=100 \mathrm{mH})$. Figure 11 shows three-phase currents before and after compensation. Figure 12 shows neutral currents before and after compensation. From Figure 11 and Figure 12, we can see that: before compensation, three-phase currents are unbalanced and asymmetrical, phase A current is with serious distortion and neutral current is also large. After compensation, three-phase currents are approximate to sine wave and the neutral current is relatively small. Figure 13 is voltage and current of the phase $\mathrm{C}$ before and after compensation, it can be seen that, voltage and current of the phase $\mathrm{A}$ are not in the same phase before compensation and with a large phase difference, however, it achieves a higher power factor and with a same frequency and phase after compensation. From the above analysis, we can see that, the APF system with one-cycle three-phase four-leg topology has many advantages of less harmonic components, less zero-sequence current and better effect of reactive power compensation ,etc..
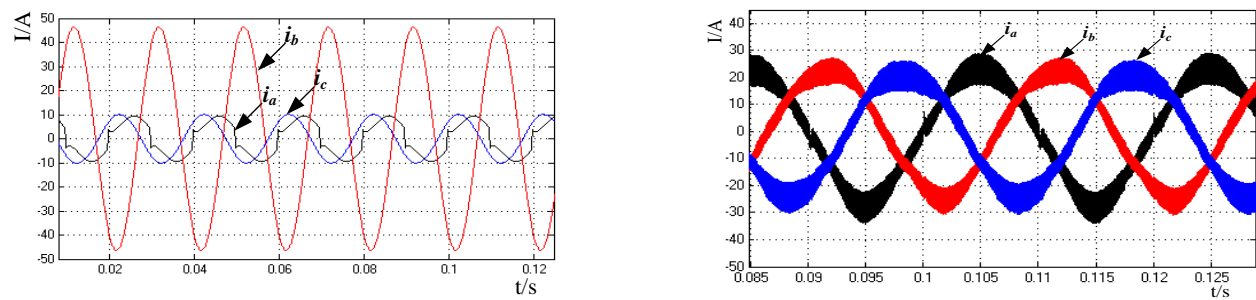

a) three-phase currents before compensation b) three-phase currents after compensation

Figure 11. Comparison of Three Phase Currents before and after Compensation

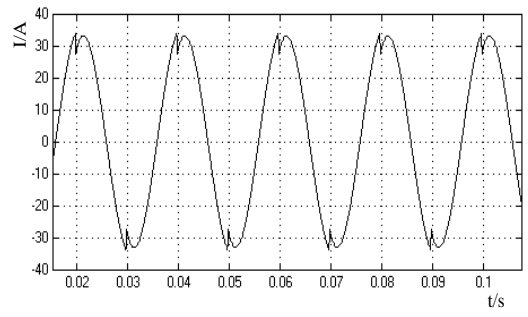

a) neutral current before compensation

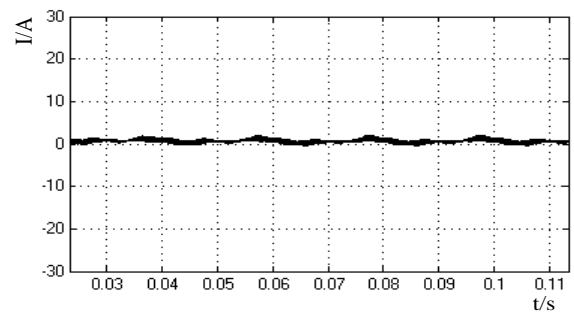

b) neutral current after compensation

Figure 12. Comparison of Neutral Current before and after compensation 


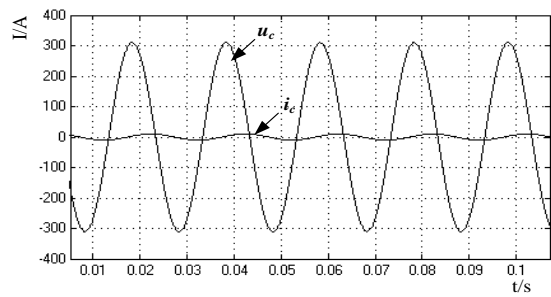

a) voltage and current of phase $\mathrm{C}$ before compensation

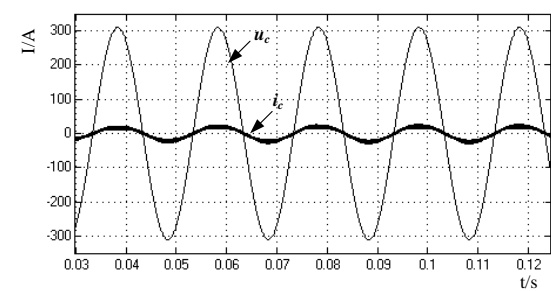

b) voltage and current of phase $\mathrm{C}$ after compensation

Figure 13. Voltage and Current of Phase $C$ before and after Compensation

(2) Three-phase unbalanced rectifier load: the unbalanced load is composed of threephase full-bridge rectifier with a resistance-inductance load $(\mathrm{R}=30 \Omega, \mathrm{L}=10 \mathrm{mH})$ and a single-phase rectifier with a resistance-inductance load $(\mathrm{R}=5 \Omega, \mathrm{L}=30 \mathrm{mH})$ which is added between phase A and neutral line. Figure 14 and Figure 15 are comparison of threephase and the neutral current relatively before and after compensation. From Figure 14 and Figure 15, it can be seen that before compensation, three-phase currents are unbalanced, with serious distortion and the neutral current is relatively large, after compensation, the three-phase current is approximate to sine wave, with small distortion and the neutral current is close to 0. Through analyzing spectrum of A and B phase current before and after compensation with fast Fourier analysis tools from Figure 16, it can be seen that the harmonic distortion decrease from $20.93 \%$ to $10.34 \%$. Figure 17 is current spectrum comparison of the phase B before and after compensation, it can be seen that the harmonic content decreases from $27.77 \%$ to $10.19 \%$. From simulation waveforms and the harmonic distortion analysis, we can see that, the APF system with one-cycle three-phase four-leg topology has many advantages of less harmonic components, less zero-sequence current and better effect of reactive power compensation ,etc..

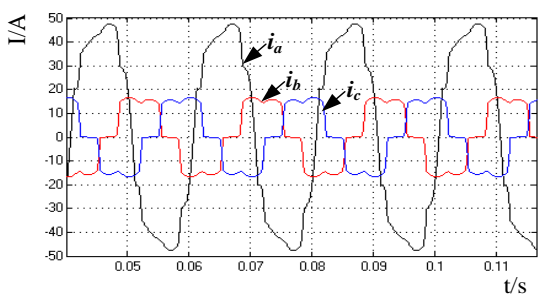

a) three phase current waveforms before compensation

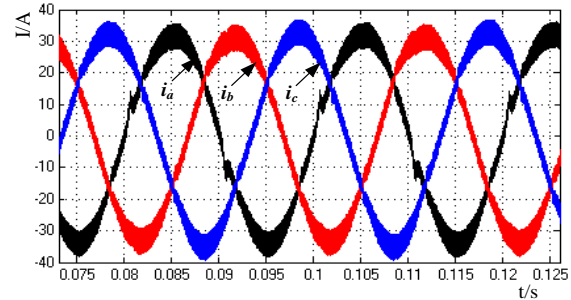

b) three phase current waveforms after compensation

Figure 14. Comparison of Three Phase Current Waveforms before and after Compensation

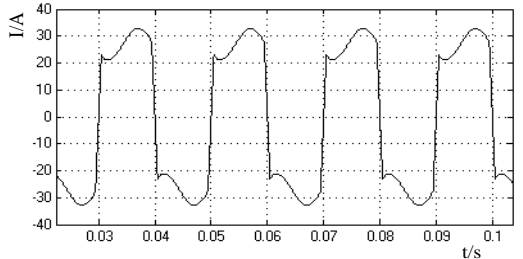

a) neutral current waveform before compensation

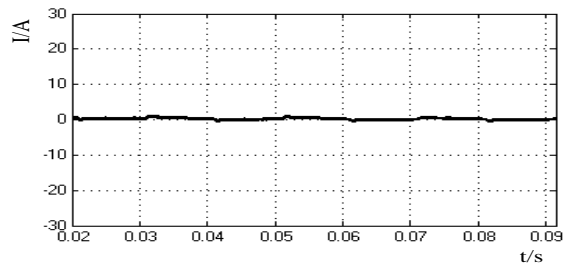

b) neutral current waveform before compensation

Figure 15. Comparison of Neutral Current Waveforms before and after Compensation 


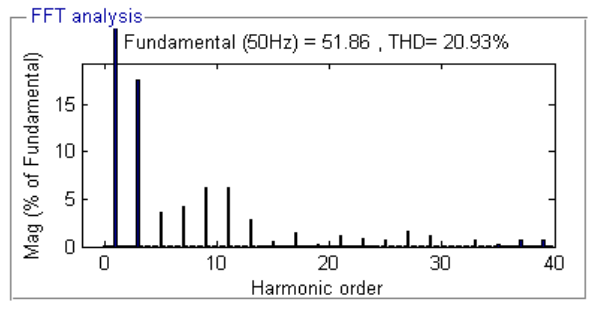

a) current spectrum of phase $A$ before compensation

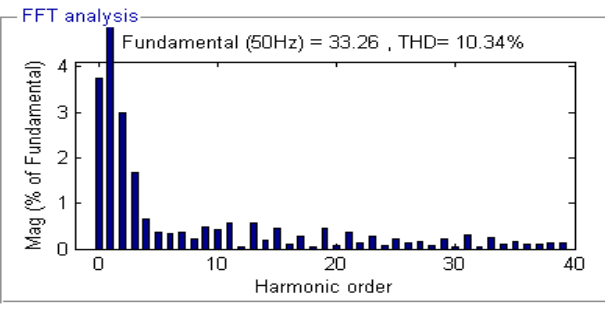

b) current spectrum of phase $A$ after compensation

Figure 16. Current Spectrum Comparison of Phase A before and after Compensation

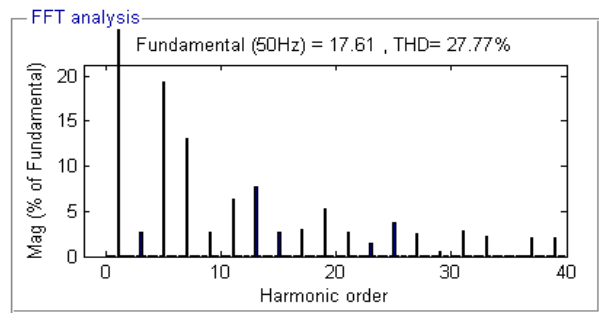

a) current spectrum of phase $B$ before compensation

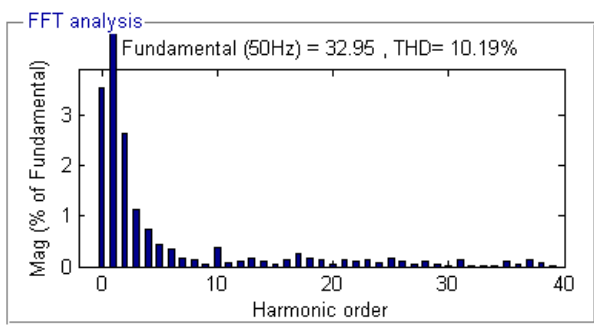

b) current spectrum of phase $B$ after compensation

\section{Figure 17. Current Spectrum Comparison of Phase B before and after} Compensation

\section{Conclusion}

In this paper, the one-cycle control technique is applied to an APF with three-phase four-leg topology. Through establishing the average switching model with continuous current mode and three-phase balanced system, the key formula of one-cycle control is derived. From integral constant analysis, this paper determines the appropriate integral constant. Based on the selected parameters, the hardware circuit is established. The system has the advantages of no multiplier in the circuit, less control variable, simple structure, low cost, fast response speed and perfect anti-interference ability, etc. From simulation results of APF with one-cycle three-phase four-leg topology, we can see that the system is capable of inhibiting harmonic, compensating zero sequence current and reactive current effectively.

\section{Acknowledgements}

This work is supported by the Hei Long Jiang postdoctoral Foundation(LBH-Z10109), HARBIN innovative talent project of science and technology(2014RFXXJ075) and teaching reform project of higher education in Hei Long Jiang Province (JG2014010795).

\section{References}

[1] Z. A. Wang and J. Yang, "Harmonic Suppression and Reactive Power Compensation", China Machine Press, Beijing (2011).

[2] D. B. Zhu, J. Liu and Y. Cui, "The Study of Active Power Filter and Static Var Generator", Journal of Harbin University of Science and Technology, vol. 7, no. 2, (2002), pp. 1-4.

[3] J. Liu, M. G. Zhang and S. Q. Xie, "Design and Simulation on The Hybrid Active Power Filter", Journal of Harbin University of Science and Technology, vol. 15, no. 5, (2010), pp. 22-26. 
[4] L. Zhou, J. W. Jiang and L. W. Zhou, "Three-phase four-wire active power filter with one-cycle control", Proceedings of the CSEE, vol. 23, no. 9, (2003), pp. 85-88.

[5] L. W. Zhou and K. M. Smedley, "Unified constant-frequency integration control of active power filters", IEEE Trans. Power Electronics, vol. 1, (2000), pp. 406-412.

[6] K. M. Smedley, L. W. Zhou and C. M. Qiao, "Unified constant- frequency integration control of active power filters-steady-state and dynamics", IEEE Trans. Power electronics, vol.16, no.3, (2001), pp. 428436.

[7] C. M. Qiao, K. M. Smedley and F. Maddaleno, "A signal-phase active power with one cycle control under unipolar operation”, IEEE Trans, vol.51, no.8, (2004), pp. 1623-1630.

[8] S. Serena, C. M. Qiao and K. M. Smedley, "A single-phase active power filter with double-edge integration control", The 27th Annual Conference of the IEEE Industrial Electronics Society, (2001), pp. 949-953.

[9] C. J. Zhang, H. R. Gu and Q. L. Zhao, "Three-phase voltage source type PWM rectifier by one-cycle control without multipliers", Transactions of China Electrotechnical Society, vol. 18, no. 6,

(2003), pp. 28-32.

[10] W. P. Zhang, "Modeling and control of switch converter", China Electrical Power Press, Beijing (2006).

[11] R. W. Wang, W. S. Cao and L. X. Chen, "Design of the boost active power factor correction circuit with one-cycle control", Electric Power Automation Equipment, vol. 31, no. 12, (2011), pp. 114-119.

[12] X. J. Zhou, "Study on one-cycle control three-phase four-wire active power filter with four-leg", Chongqing: Chongqing university, (2003).

[13] X. Du, L. W. Zhou and Q. M. Luo, "The effect of integration time constant of one cycle controlled threephase power factor correction", Proceedings of the CSEE, vol. 26, no. 9, (2006).

[14] B. Liu, "Development of Three-phase Four-leg SVG", Harbin: Harbin University of Science and Technology, (2013).

[15] X. Du, "Study on one cycle controlled DC side active power filter", Chongqing: Chongqing university, (2002). 